\title{
Predictors of Acute Kidney Injury in Geriatric Patients Undergoing Total Knee Replacement Surgery
}

\author{
Vishal Sehgal ${ }^{1}$; Sukhminder Jit Singh Bajwa ${ }^{2, *}$; Rinku sehgal ${ }^{3}$; Jeremiah Eagan ${ }^{4}$; Praveen \\ Reddy $^{3}$; Samuel M. Lesko ${ }^{5}$ \\ ${ }^{1}$ Department of Internal Medicine, University of South Carolina.Greenville, South Carolina, USA \\ ${ }_{3}^{2}$ Department of Anesthesiology and Intensive Care Medicine, Gian Sagar Medical College, Banur, Patiala, Punjab, India \\ 3 Department of Internal Medicine, Wright Center, Scranton, USA \\ 4 Department of Neprology, Regional Hospital, Scranton, USA \\ ${ }^{5}$ Dortheast Regional Cancer Institute, University of Scranton, Scranton, USA \\ ${ }^{*}$ Corresponding author: Sukhminder jit Singh Bajwa, Department of Anesthesiology and Intensive Care Medicine, Gian Sagar Medical College, Banur, Patiala, Punjab, India. Tel: +99- \\ 15025828, +91-1752352182, Fax:+86-47976115, E-mail: sukhminder_bajwa2001@yahoo.com
}

Received: January 4, 2014; Revised: March 16, 2014; Accepted: March 22, 2014

\begin{abstract}
Background: Few studies have focused on patients' characteristics that affects acute kidney injury (AKI) after total knee replacement surgery (TKR).

Objectives: The primary objective of this retrospective study was to identify patients' characteristics associated with AKI after TKR.

Patients and Methods: Between January 2008 and December 2009, 659 patients with a mean age of 67.1 years (range, 39-99) underwent TKA at Regional Hospital Knee and Hip Institute. Retrospective chart review was done to identify patients' characteristics that were associated with AKI after TKR. Logistic regression was used to evaluate AKI.

Results: AKI occurred in 21.9\% of patients. AKI risk decreased between 2008 and 2009 (odds ratio, 0.55; 95\% CI, -0.37 to 0.82 ) but increased with age $(\mathrm{P}<0.001)$, diabetes mellitus $(\mathrm{DM})$, and angiotensin converting enzyme inhibitors (ACEI)/angiotensin receptor blockers (ARB) use (OR, -1.6; 95\% CI, -1.0 to 2.5; and OR, -1.5, 95\% CI, -1.0 to 2.3, respectively). However, the effects of DM and ACEI/ARB use were not independent; when both were included in the regression model, neither was statistically significant, and both ORs were smaller than combined OR. Conclusions: When examined separately, both DM and preoperative ACEI/ARB use increased the risk of AKI; however these factors were correlated and were not independent predictors of significantly increased risk. Patients with DM have higher tendency to develop AKI and hence, preoperative renal risk stratification should be done in all patients with DM.
\end{abstract}

Keywords:Acute Kidney Injury; Geriatrics; Chronic Kidney Disease; Diabetes Mellitus; Total Knee Replacement; Health Care Reform

\section{Background}

In the light of ever increasing geriatric population, healthcare budgets are constrained. Medicare is expected to cut down healthcare reimbursements in the years to come. The healthcare services would become more and more competitive. This is in sharp contrast to earlier trends when hospitals were reimbursed irrespective of the quality of the services offered. In addition, the definition of quality needs to be redefined as it is not only increased utilization of healthcare resources but also improved outcomes at lower costs. Increasingly, we see aging of the population in the $21^{\text {st }}$ century with the fastest growing population being people over 85 years of age. With the baby boomers moving into geriatric age group, this section of population is going to get even larger. With the aging of the population, more patients with chronic kidney disease (CKD) undergo elective surgeries. Perioperative risk stratification focuses on optimizing the cardiopulmonary function. There is little or no emphasis on renal risk stratification and renal protection strategies $(1,2)$. With aging, renal function declines significantly. Peak body mass with optimum body physiology is achieved by the age of 30 years; thereafter, there is a gradual decline in it. As the person moves from 30 to 80 years of age, usually $30 \%$ of the kidney function is lost (3). The predominant loss is in the renal cortex that affects the most physiologically important nephrons. It results in about $40 \%$ lower renal plasma flow in the elderly as compared to the younger adults even when there is no risk factor for kidney disease. A deeper study of the renal diseases based on molecular genetics can help to

\section{Implication for health policy/practice/research/medical education}

Implication for health policy/practice/research/medical education

Diabetes mellitus (DM) and preoperative angiotensin converting enzyme inhibitors (ACEI) use increased the risk of acute kidney injury(AKI); however, these factors were correlated and were not independent predictors of significantly increased risk of AKI. Patients with DM tend to develop AKI more than general population and hence, there is need for meticulous preoperative renal riskstratification.

Copyright (c) 2014, Research Institute For Endocrine Sciences and Iran Endocrine Society; Published by Kowsar Corp. This is an open-access article distributed under the terms of the Creative Commons Attribution License, which permits unrestricted use, distribution, and reproduction in any medium, provided the original work is properly cited. 
develop a better knowledge and understanding of these facts $(4,5)$. To compensate for it, the elderly's kidneys are maintained in a state of chronic vasodilatation. In perioperative settings, it contributes to increase risk of renal injury in the elderly population especially the septuagenarians and octogenarians. Moreover, when renal injury does occur, the elderly are less likely to recover, especially after an ischemic insult to the kidneys (6-8).

Acute kidney injury (AKI) has traditionally been defined as an abrupt loss of kidney function with resultant accumulation of nitrogenous waste and dysregulation of electrolytes and blood volume. The loss of kidney function may most easily be quantified by measuring the serum creatinine $(\mathrm{Cr})$. It has its own pitfalls as in the elderly who usually have decreased muscle mass, the rise of serum $\mathrm{Cr}$ is not proportionate to the loss of kidney function. $\mathrm{Cr}$ production decreases with aging; in addition, there is decrease in tubular secretion of $\mathrm{Cr}$. Hence, despite significant decrease in glomerular filtration rate (GFR) in the elderly, Cr may remain within normal range (9). The prevalence of CKD is increased by increased incidence of AKI (10). Moreover, when patients with underlying CKD are exposed to potentially nephrotoxic medications or physiological conditions, they are at increased risk of AKI in the postoperative period. The incidence of such injuries is increased in highrisk patients admitted to the ICUs with comorbidities. This is true even for stage I CKD, which is defined by proteinuria with normal GFR (11-14). In the elderly, AKI is an independent risk factor for hospital mortality and is associated with longer ICU stay, increased morbidity, utilization of resources, and higher mortality at six-month follow-up (15-20). In addition, it is an important prognostic marker for complications during hospitalization in the elderly (21).

Total hip and total knee replacement surgeries (THR and TKR, respectively) for osteoarthritis (OA) are amongst the most successful orthopedic interventions with a positive impact on health-related quality of life. There will be a considerable increase in the number of joint replacement surgeries, even in the low income countries, in the years to come. Physicians will be frequently involved in the medical consultations with patients in the postoperative period of THR/TKR. Current data suggest that postoperative AKI is associated with increased hospital length of stay (22). It leads to loss of revenue to the hospital and decreased patient satisfaction after the elective surgery. We have been seeing substantial AKI in this subset of population. Most of the data on perioperative AKI are from patients with cardiac surgeries (23).

\section{Objectives}

The present study aimed to identify the patients' characteristics associated with AKI. Diabetes status and angiotensin converting enzyme inhibitors (ACEI) as well as angiotensin receptor blocker (ARB) usage among the elderly patients were of particular interest.

\section{Patients and Methods}

The present study was conducted at a specialty surgical service of a community-based acute care hospital. All patients who underwent TKR surgeries between January 2008 and December 2009, were included in the study regardless of their age, sex, race, comorbidities, and socioeconomic status. AKI was defined as an abrupt (within 48 hours) absolute increase in the serum $\mathrm{Cr}$ concentration of $\geq 0.3 \mathrm{mg} / \mathrm{dL}$ from baseline. This was based on Acute Kidney Injury Network (AKIN) criteria for AKI (24). Using AKIN, AKI was further subdivided into stages I through III: Stage I, $\geq 0.3 \mathrm{mg} / \mathrm{dL}$ increase in serum; Stage II, two to 2.9 times the baseline increase in the serum $\mathrm{Cr}$; and stage III, three times the baseline increase in the serum Cr. Urine output was not measured. Included secondary characteristics were age, sex, and marital status. The design of the study was presented to the Institutional Review Board (IRB). The contents of the study included the patients' characteristics and associated comorbidities. After going through the design of the study, the IRB waived the requirement for informed consent as this was a retrospective review of medical records. Demographic and laboratory data were retrieved from an electronic hospital database. After merging data from the different sources, automated and manual data verification was performed.

\subsection{Statistical Analysis}

All events were recorded from the day of surgery up to the discharge from the acute care hospital. Continuous variables were compared using the independent samples t test and categorical data were compared with the chi square $\left(\chi^{2}\right)$ test. Logistic regression was used to evaluate the association between patients' characteristics and AKI while controlling for confounding variables. The logistic model included terms for age, sex, year of surgery, DM, and ACEI use. In all tests, P value $<0.05$ was considered as statistically significant finding.

\section{Results}

During the study period, a total of 659 (442 females) patients underwent TKR. Of these, 32 were excluded because of missing or incomplete data precluded assessment of renal status. The analysis was limited to 627 patients with sufficient data to evaluate AKI. The characteristics of these patients are shown in Table 1. Overall, 137 (21.9\%) patients experienced an episode of AKI (Figure 1) following surgery (stage I, 120; stage II, 17; and no stage III). Patients with AKI were older (mean age, $71.1 \pm 10.2$ years) than those without AKI (mean age, $66.1 \pm 10.2$ years; $\mathrm{P}<0.001$ ). Risk of AKI did not differ by sex; however, risk of AKI was greater in 2008 than in 2009 ( $\mathrm{P}=0.002)$. In addition, risk of AKI was greater among patients with DM and those exposed to ACEIs. Risk of AKI increased by 5\% per year of life and, compared to 2008 , was approximately $45 \%$ lower in 2009 . In the bivariate analysis, the risk of AKI was significantly elevated among patients with both DM (OR, 1.58; 95\% CI, 1.04-2.40) 
Sehgal Vet al.

\begin{tabular}{|c|c|c|c|}
\hline Characteristic & $\operatorname{AKI}(n=137)$, No. $(\%)$ & No AKI (n= 490), No. (\%) & PValue \\
\hline Age, mean \pm SD & $71.1 \pm 10.2$ & $66.1 \pm 10.2$ & $<0.001$ \\
\hline Sex & & & NS \\
\hline Female & $85(20.3)$ & $334(79.7)$ & \\
\hline Male & $52(25.0)$ & $156(75.0)$ & \\
\hline Year & & & 0.002 \\
\hline 2008 & $79(27.3)$ & $210(72.7)$ & \\
\hline 2009 & $58(17.2)$ & $280(82.8)$ & \\
\hline DM & & & 0.031 \\
\hline Yes & $43(28.1)$ & $110(71.9)$ & \\
\hline No & $94(19.8)$ & $380(80.2)$ & \\
\hline ACEI/ARB use $\mathrm{b}^{\mathrm{b}}$ & & & 0.028 \\
\hline Any & $55(27.0)$ & $149(73.0)$ & \\
\hline None & $81(19.2)$ & $340(80.0)$ & \\
\hline
\end{tabular}

a Abbreviations: AKI, acute kidney injury; NS, nonsignificant; DM, diabetes mellitus; ACEI, angiotensin converting enzyme inhibitors; ARB, angiotensin receptor blocker.

b Patients with unknown ACEI exposure (one with AKI, one without AKI) were excluded.

Table 2. Frequency of Acute Kidney Injury Stages Among the Participants and Mutually Exclusive Categories of Diabetes and angiotensin converting enzyme inhibitors Exposure ${ }^{\mathrm{a}, \mathrm{b}}$

\begin{tabular}{lccc}
\hline \multirow{2}{*}{ Exposure } & \multicolumn{2}{c}{ AKI } & \multirow{2}{*}{ No AKI } \\
\cline { 2 - 3 } & Stage I & Stage II & \\
\hline Total & $120(19.1)$ & $17(2.7)$ & $490(78.1)$ \\
None & $59(16.7)$ & $7(2.0)$ & $288(81.4)$ \\
DM Alone & $13(19.4)$ & $2(3.0)$ & $52(77.6)$ \\
ACEI Use Alone & $25(21.0)$ & $3(2.5)$ & $91(76.5)$ \\
DM + ACEI Use & $22(25.9)$ & $5(5.9)$ & $58(68.2)$ \\
Unknown ACEI Exposure & $1(50)$ & $0(-)$ & $1(50)$ \\
\hline
\end{tabular}

${ }^{a}$ Abbreviations: AKI, acute kidney injury; DM, diabetes mellitus; ACEI, angiotensin converting enzyme inhibitors.

${ }^{b}$ Data are presented as No.(\%).

Figure 1. Incidence of Acute Kidney Injury in the Study

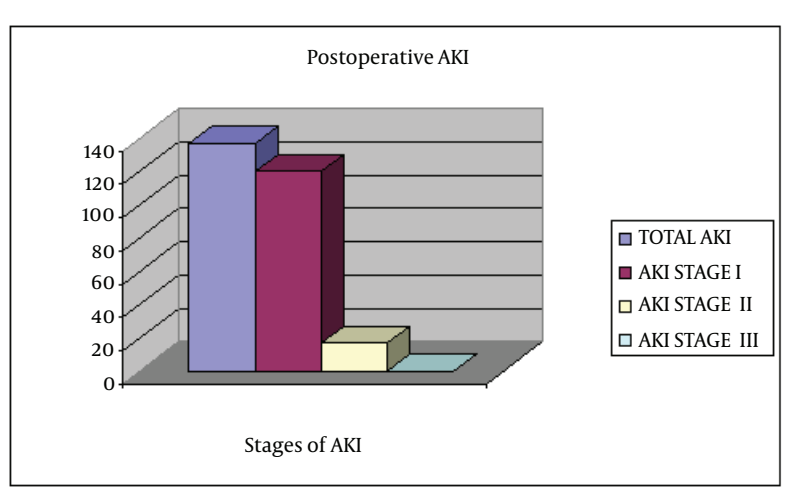

Acute kidney injury (AKI) was seen in 138 patients. Of these, 120 and 18 had AKI stages I and II, respectively. There was no patient with AKI stage III. and ACEI use (OR, 1.55; 95\% CI, 1.05-2.30); the risk was greatest among patients with DM who used ACEI (OR, 2.03; 95\% CI:1.20- 3.45). In the multivariable model, mutually exclusive terms were used to indicate patients with DM only (no ACEI use), ACEI use only (without DM), and both DM and ACEI use. In this model, in comparison to patients with neither DM nor ACEI use, the risk of AKI was not significantly elevated in patients with DM alone or those with only ACEI use; however, the risk was nearly two-fold elevated among patients with DM who had also used ACEI (OR, 1.91; 95\% CI, 1.10-3.32). The distribution of patients according to the stage of AKI and mutually exclusive categories of DM and ACEI use is shown in Table 2. Risk of AKI was greatest among the 85 patients with DM who used preoperative ACEI, 27 (31.8\%) of whom experienced AKI following surgery. Compared to patients without DM or ACEI use, the risk of stage II AKI was nearly three-fold greater among patients with DM who used ACEIs $(\mathrm{P}<0.05)$. The number of stage II AKI patients was very small for multivariable analysis.

\section{Discussion}

With the recent trend towards minimally invasive total joint arthroplasty and the increased emphasis on faster recovery and shorter hospital stays, it has become increasingly important to recognize the timing and severity of the various complications associated with elective total joint arthroplasty. Complications following TKR usually occur within the time frame of the typical hospital stay. AKI is a complication that occurs frequently in hospitalized patients. The incidence of AKI in the hospitalized elderly has increased over the past decade and is associated with a substantial increase in morbidity and mortality (25-27). Renal impairment is significantly as- 
sociated with lengthened duration of hospital stay and one-year all-cause mortality $(22,28,29)$. Long-term mortality is greater in patients who survived AKI in comparison to critically or non-critically ill patients without AKI $(30,31)$. Outcomes are related directly to the severity of AKI, whether characterized by nominal or percentage changes in serum $\mathrm{Cr}$. The higher the duration and stage of AKI are, the higher the long-term morbidity and mortality rates will be (32).

Age, high-risk surgery, DM, metabolic syndrome, use of radiographic contrasts, peripheral vascular disease, alternative medicine drugs, perioperative blood transfusions, general anesthesia, HIV infection, ischemic heart disease, diuretic use, and congestive heart disease are considered risk factors for the development of AKI (16, 3344). In all the multivariate studies evaluating the risk factors for AKI, age has been consistently seen as potential risk factor (41). In our study, there was a wide variation in the extent of AKI depending on the age. Patients older than 85 years of age were more likely to have severe AKI. In contrast, patients older than 65 years were more likely to develop mild AKI. Obesity and oxidative stress are also independent predictors of AKI $(30,45)$. Moreover, some ethnic groups are more predisposed to AKI than others (46). This retrospective study showed a statistically significant association between preoperative comorbidities and postoperative AKI in elective TKR surgeries. The incidence of AKI is variable depending on whether the patient has DM or is currently on ACEI or both (34). There is a direct association with AKI if the patient has DM and is on ACEI. DM or ACEI by themselves were not directly implicated in the pathogenesis of AKI.

In our study, the AKI was relatively mild with detecting only AKI stage I and II and no stage III in patients. The perioperative risk stratification could be helpful in anticipating at risk population who could develop AKI after major surgeries (16). Adoption of perioperative renal protection strategies can be extremely helpful in all the patients, including the high-risk ones, undergoing TKR and other joint replacement surgeries. Diagnosis of AKI in the hospitalized elderly can be tricky. All the criteria defining AKI are based on Cr; however, in elderly with low muscle mass, there may be surprisingly slow increase in serum Cr. Novel biomarkers of the tubulointerstitial injury akin to the ones used in myocardial infarction would make the diagnosis of AKI easier in the elderly $(41,47)$.

This study had some limitations. The factors other than DM, age, and ACEI use, which could possibly precipitate acute renal failure, could not be controlled; however, we believe that factors such as intraoperative hypotension might be a consequence of ACEI use in patients with DM rather than a simple confounder. In addition, we used AKIN to diagnose and quantify AKI in the postoperative settings. AKIN may lead to overdiagnosis of AKI in the elderly where a rise in serum $\mathrm{Cr}$ is not corrected for fluid balance in the postoperative settings (48). Therapeutic intervention and renal replacement therapy have mini- mal impact on the AKI. Resources should focus on renal risk stratification and preventing AKI in the elderly in the perioperative settings $(41,49,50)$. Clinicians should be able to anticipate AKI on the basis of patients' clinical characteristics and minimize the morbidities occurring with postoperative renal failure (51). Strategies should also include modifications in the techniques of anesthesia and titration in addition to practicing renal protection techniques during perioperative period (2, 52-54).

Onuigbo et al. stated that for renal protection in the elderly with underlying CKD, it is reasonable to discontinue ACEI/ARB two to four days prior to elective surgeries or invasive procedures with risk of AKI (55). The same seems to be correct in the elderly who are undergoing elective joint surgeries and are at risk for AKI. In addition, preoperative screening should assess the underlying CKD and risk factors that predisposes to perioperative AKI. Euvolemia should be maintained and all nephrotoxic medications should be preferably avoided in this population $(2,56,57)$. Use of preoperative statins as a means to reduce AKI is still under examination and needs further assessment to make any firm recommendations (58). Multidisciplinary approach in which the tandem of the surgeon, anesthesiologist, and internist approach the patient to minimize the perioperative AKI would be reasonable.

In conclusion, we report a retrospective cohort study which reinforces the need for perioperative renal risk stratification especially in the elderly population with DM. This would be cost-effective and would improve morbidity and mortality associated with AKI in the elderly. Further prospective, randomized, double blind studies are needed to substantiate these findings further.

\section{Acknowledgements}

The authors expressed their sincere thanks to the staff of the hospital.

\section{Authors' Contributions}

Study concept and design: Vishal Sehgal, Sukhminder Jit Singh Bajwa, Rinku Sehgal, Praveen Reddy, Jeremiah Eagan, Samuel M. Lesko, and Victoria Kresse. Acquisition of data: Vishal Sehgal, Rinku Sehgal and Praveen Redd. Acquisition of Literature: Vishal Sehgal, Sukhminder Jit Singh Bajwa, Rinku Sehgal, Victoria Kresse, and Praveen Reddy. Analysis and interpretation of data: Vishal Sehgal, Sukhminder Jit Singh Bajwa, Rinku Sehgal, Praveen Reddy, Jeremiah Eagan, Samuel M. Lesko, and Victoria Kresse. Drafting of the manuscript: Vishal Sehgal, Sukhminder Jit Singh Bajwa, Rinku Sehgal, Praveen Reddy, Jeremiah Eagan, Samuel M. Lesko, and Victoria Kresse. Critical revision of the manuscript for important intellectual content: Vishal Sehgal, Sukhminder Jit Singh Bajwa, Rinku Sehgal, Praveen Reddy, Jeremiah Eagan, Samuel M. Lesko, and Victoria Kresse. Statistical analysis: Vishal Sehgal, Sukhminder Jit Singh Bajwa, Rinku Sehgal, Praveen Red- 
dy, Jeremiah Eagan, Samuel M. Lesko, and Victoria Kresse. Administrative, technical, and material support: Vishal Sehgal, Rinku Sehgal. Praveen Reddy, Jeremiah Eagan, Samuel M. Lesko, and Victoria Kresse. Study supervision: Vishal Sehgal.

\section{Financial Disclosure}

We had no financial interests related to the material in the manuscript.

\section{Funding/Support}

No support from any source was received.

\section{References}

1. Brienza N, Giglio MT, Marucci M. Preventing acute kidney injury after noncardiac surgery. Curr Opin Crit Care. 2010;16(4):353-8.

2. Bajwa SJ, Sharma V. Peri-operative renal protection: The strategies revisited. Indian J Urol. 2012;28(3):248-55.

3. Nyengaard JR, Bendtsen TF. Glomerular number and size in relation to age, kidney weight, and body surface in normal man. Anat Rec. 1992;232(2):194-201.

4. Fuiano G, Sund S, Mazza G, Rosa M, Caglioti A, Gallo G, et al. Renal hemodynamic response to maximal vasodilating stimulus in healthy older subjects. Kidney Int. 2001;59(3):1052-8.

5. Bajwa SJ, Kwatra IS. Reno-endocrinal disorders: A basic understanding of the molecular genetics. Indian J Endocrinol Metab. 2012;16(2):158-63.

6. Ried M, Puehler T, Haneya A, Schmid C, Diez C. Acute kidney injury in septua- and octogenarians after cardiac surgery. BMC Cardiovasc Disord. 2011;11:52.

7. Schmitt R, Cantley LG. The impact of aging on kidney repair. Am J Physiol Renal Physiol. 2008;294(6):F1265-72.

8. Bajwa SJ, Kulshrestha A. Renal endocrine manifestations during polytrauma: A cause of concern for the anesthesiologist. Indian J Endocrinol Metab. 2012;16(2):252-7.

9. Giannelli SV, Patel KV, Windham BG, Pizzarelli F, Ferrucci L, Guralnik JM. Magnitude of underascertainment of impaired kidney function in older adults with normal serum creatinine. J Am Geriatr Soc. 2007;55(6):816-23.

10. Cho A, Lee JE, Kwon GY, Huh W, Lee HM, Kim YG, et al. Post-operative acute kidney injury in patients with renal cell carcinoma is a potent risk factor for new-onset chronic kidney disease after radical nephrectomy. Nephrol Dial Transplant. 2011;26(11):3496-501.

11. Moore EM, Bellomo R, Nichol AD. The meaning of acute kidney injury and its relevance to intensive care and anaesthesia. Anaesth Intensive Care. 2012;40(6):929-48.

12. Bajwa SS, Kwatra IS. Nutritional needs and dietary modifications in patients on dialysis and chronic kidney disease. JMed Nutr Nutraceut. 2013;2(1):46-51.

13. Hsu RK, Hsu CY. Proteinuria and reduced glomerular filtration rate as risk factors for acute kidney injury. Curr Opin Nephrol Hypertens. 2011;20(3):211-7.

14. Bajwa SJ, Singh K, Bajwa SK, Singh A, Singh G, Panda A. Anesthesia considerations in a" very old" geriatric patient for major orthopedic surgery. Anesth Essays Res. 2010;4(2):125-6.

15. Ulucay C, Eren Z, Kaspar EC, Ozler T, Yuksel K, Kantarci G, et al. Risk factors for acute kidney injury after hip fracture surgery in the elderly individuals. Geriatr Orthop Surg Rehabil. 2012;3(4):150-6.

16. Weingarten TN, Gurrieri C, Jarett PD, Brown DR, Berntson NJ, Calaro RJ, et al. Acute kidney injury following total joint arthroplasty: retrospective analysis. Can JAnaesth. 2012;59(12):1111-8.

17. Li SY, Chen JY, Yang WC, Chuang CL. Acute kidney injury network classification predicts in-hospital and long-term mortality in patients undergoing elective coronary artery bypass grafting surgery. EurJ Cardiothorac Surg. 2011;39(3):323-8.

18. Abelha FJ, Botelho M, Fernandes V, Barros H. Determinants of postoperative acute kidney injury. Crit Care. 2009;13(3):R79.
19. Bihorac A, Yavas S, Subbiah S, Hobson CE, Schold JD, Gabrielli A, et al. Long-term risk of mortality and acute kidney injury during hospitalization after major surgery. Ann Surg. 2009;249(5):851-8.

20. Jaschinski U, Lichtwarck-Aschoff M. [Acute perioperative disturbances of renal function. Strategies for prevention and therapy]. Anaesthesist. 2009;58(8):829-47.

21. De Santo LS, Romano G, Amarelli C, Maiello C, Baldascino F, Bancone C, et al. Implications of acute kidney injury after heart transplantation: what a surgeon should know. EurJ Cardiothorac Surg. 2011;40(6):1355-61.

22. Noor S, Usmani A. Postoperative renal failure. Clin Geriatr Med. 2008;24(4):721-9.

23. Thakar CV. Perioperative acute kidney injury. Adv Chronic Kidney Dis. 2013;20(1):67-75.

24. Mehta RL, Kellum JA, Shah SV, Molitoris BA, Ronco C, Warnock DG, et al. Acute Kidney Injury Network: report of an initiative to improve outcomes in acute kidney injury. Crit Care. 20 07;11(2):R31.

25. Gong ZY, Gao CQ, Li B], Jiang SL, Xiao CS, Wang R, et al. [Acute kidney injury early after cardiac surgery with cardiopulmonary bypass: clinical analysis]. Zhonghua Yi Xue Za Zhi. 2012;92(46):3283-7.

26. Olsson D, Sartipy U, Braunschweig F, Holzmann MJ. Acute kidney injury following coronary artery bypass surgery and long-term risk of heart failure. Circ Heart Fail. 2013;6(1):83-90.

27. Hoste EA, Kellum JA, Katz NM, Rosner MH, Haase M, Ronco C. Epidemiology of acute kidney injury. Contrib Nephrol. 2010;165:1-8.

28. Kiers HD, van den Boogaard M, Schoenmakers MC, van der Hoeven JG, van Swieten HA, Heemskerk S, et al. Comparison and clinical suitability of eight prediction models for cardiac surgery-related acute kidney injury. Nephrol Dial Transplant. 2013;28(2):345-51.

29. Wu VC, Huang TM, Lai CF, Shiao CC, Lin YF, Chu TS, et al. Acuteon-chronic kidney injury at hospital discharge is associated with long-term dialysis and mortality. Kidney Int. 2011;80(11):1222-30.

30. Bajwa SJ, Sehgal V, Bajwa SK. Clinical and critical care concerns in severely ill obese patient. Indian J Endocrinol Metab. 2012;16(5):740-8.

31. Cantaluppi V, Quercia AD, Dellepiane S, Figliolini F, Medica D, De Lena M. [New mechanisms and recent insights in the pathogenesis of acute kidney injury (AKI)]. G Ital Nefrol. 2012;29(5):535-47.

32. Coca SG, King JJ, Rosenthal RA, Perkal MF, Parikh CR. The duration of postoperative acute kidney injury is an additional parameter predicting long-term survival in diabetic veterans. Kidney Int. 2010;78(9):926-33.

33. Brown JR, Kramer RS, MacKenzie TA, Coca SG, Sint K, Parikh CR. Determinants of acute kidney injury duration after cardiac surgery: an externally validated tool. Ann Thorac Surg. 2012;93(2):570-6.

34. Bajwa SJ, Kalra S. Diabeto-anaesthesia: A subspecialty needing endocrine introspection. Indian J Anaesth. 2012;56(6):513-7.

35. Xu JR, Teng J, Fang Y, Shen B, Liu ZH, Xu SW, et al. [The risk factors and prognosis of acute kidney injury after cardiac surgery: a prospective cohort study of 4007 cases]. Zhonghua Nei Ke Za Zhi. 2012;51(12):943-7.

36. van Kuijk JP, Flu WJ, Valentijn TM, Chonchol M, Voute MT, Kuiper RJ, et al. Preoperative left ventricular dysfunction predisposes to postoperative acute kidney injury and long-term mortality. $J$ Nephrol. 2011;24(6):764-70.

37. Bajwa SJ, Panda A. Alternative medicine and anesthesia: Implications and considerations in daily practice.Ayu. 2012;33(4):475-80.

38. Weir MR, Aronson S, Avery EG, Pollack CJ. Acute kidney injury following cardiac surgery: role of perioperative blood pressure control. Am J Nephrol. 2011;33(5):438-52.

39. Brown JR, Robb JF, Block CA, Schoolwerth AC, Kaplan AV, O'Connor GT, et al. Does safe dosing of iodinated contrast prevent contrast-induced acute kidney injury? Circ Cardiovasc Interv. 2010;3(4):346-50.

40. Bajwa SJ, Kulshrestha A. The potential anesthetic threats, challenges and intensive care considerations in patients with HIV infection. J Pharm Bioallied Sci. 2013;5(1):10-6.

41. Chronopoulos A, Cruz DN, Ronco C. Hospital-acquired acute kidney injury in the elderly. Nat Rev Nephrol. 2010;6(3):141-9.

42. Glance LG, Wissler R, Mukamel DB, Li Y, Diachun CA, Salloum R, et al. Perioperative outcomes among patients with the modified 
metabolic syndrome who are undergoing noncardiac surgery. Anesthesiology. 2010;113(4):859-72.

43. Kochi AC, Martins AS, Lima MC, Martin LC, Balbi AL. [Preoperative factors for the development of acute kidney injury after cardiac surgery: prospective study]. Rev Assoc Med Bras. 2008;54(3):20813.

44. Lombardi R, Ferreiro A. Risk factors profile for acute kidney injury after cardiac surgery is different according to the level of baseline renal function. Ren Fail. 2008;30(2):155-60.

45. Billings F, Pretorius M, Schildcrout JS, Mercaldo ND, Byrne JG, Ikizler TA, et al. Obesity and oxidative stress predict AKI after cardiac surgery. J Am Soc Nephrol. 2012;23(7):1221-8.

46. Chew ST, Mar WM, Ti LK. Association of ethnicity and acute kidney injury after cardiac surgery in a South East Asian population. BrJAnaesth. 2013;110(3):397-401.

47. Haase M, Story DA, Haase-Fielitz A. Renal injury in the elderly: diagnosis, biomarkers and prevention. Best Pract Res Clin Anaesthesiol. 2011;25(3):401-12.

48. Englberger L, Suri RM, Li Z, Casey ET, Daly RC, Dearani JA, et al. Clinical accuracy of RIFLE and Acute Kidney Injury Network (AKIN) criteria for acute kidney injury in patients undergoing cardiac surgery. Crit Care. 2011;15(1):R16.

49. Coleman MD, Shaefi S, Sladen RN. Preventing acute kidney injury after cardiac surgery. Curr Opin Anaesthesiol. 2011;24(1):70-6.

50. Zhang W, Shen PY, Chen YX, Zhu P, Shi H, Li X, et al. Analyzing Chi- nese patients with post-operative acute kidney injury. Ren Fail. 2011;33(8):795-800.

51. Kateros K, Doulgerakis C, Galanakos SP, Sakellariou VI, Papadakis SA, Macheras GA. Analysis of kidney dysfunction in orthopaedic patients. BMC Nephrol. 2012;13:101.

52. Kumar S, Bajwa SJ. Neuraxial opioids in geriatrics: A dose reduction study of local anesthetic with addition of sufentanil in lower limb surgery for elderly patients. Saudi $J$ Anaesth. 2011;5(2):142-9.

53. Singh Bajwa SJ, Bajwa SK, Kaur J. Comparison of two drug combinations in total intravenous anesthesia: Propofol-ketamine and propofol-fentanyl. Saudi J Anaesth. 2010;4(2):72-9.

54. Bajwa SJ, Kalra S. Logical empiricism in anesthesia: A step forward in modern day clinical practice. J Anaesthesiol Clin Pharmacol. 2013;29(2):160-1.

55. Onuigbo MA. Can ACE inhibitors and angiotensin receptor blockers be detrimental in CKD patients? Nephron Clin Pract. 2011;118(4):c407-19.

56. Pucelikova T, Dangas G, Mehran R. Contrast-induced nephropathy. Catheter Cardiovasc Interv. 2008;71(1):62-72.

57. Jones DR, Lee HT. Perioperative renal protection. Best Pract Res Clin Anaesthesiol. 2008;22(1):193-208.

58. Argalious MY, Dalton JE, Sreenivasalu T, O'Hara J, Sessler DI. The association of preoperative statin use and acute kidney injury after noncardiac surgery. Anesth Analg. 2013;117(4):916-23. 